\title{
GSM VE ISM BANDI RF ENERJİ HASATLAMA SİSTEMLERİ İÇIN TEK KATLI DOĞRULTUCU DEVRE TASARIMI
}

\author{
Aysu BELEN ${ }^{*}$, Mehmet Ali BELEN ${ }^{2}$ \\ 1 İskenderun Teknik Üniversitesi, İskenderun Meslek Yüksek Okulu, Hibrid ve Elektrikli Araçlar Bölümü, Hatay, \\ Türkiye \\ 2 İskenderun Teknik Üniversitesi, Mühendislik ve Doğa Bilimleri Fakültesi, Elektrik Elektronik Mühendisliği \\ Bölümü, Hatay, Türkiye
}

\begin{tabular}{|c|c|}
\hline Anahtar Kelimeler & Öz \\
\hline $\begin{array}{l}\text { RF Enerji Hasatlama, } \\
\text { Kablosuz Enerji Transferi, } \\
\text { GSM, } \\
\text { ISM, } \\
\text { SMS7630. }\end{array}$ & 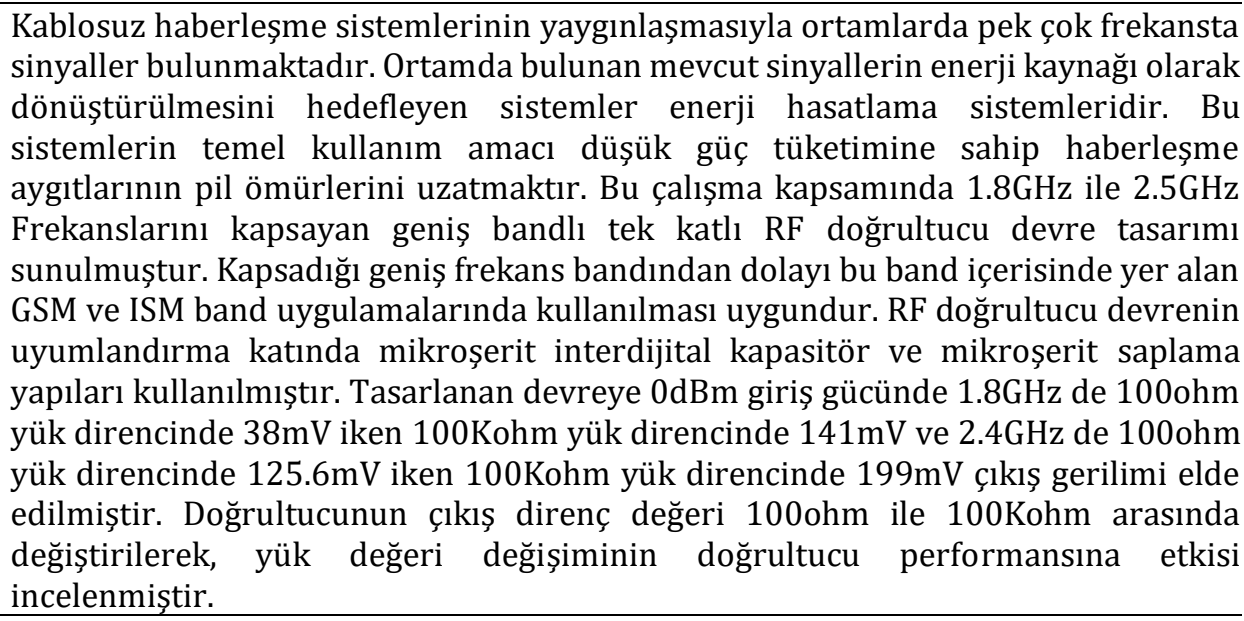 \\
\hline
\end{tabular}

\section{SINGLE STAGE RECTIFIER CIRCUIT DESIGN FOR GSM AND ISM BAND RF ENERGY HARVESTING SYSTEMS}

\section{Keywords}

RF Energy Harversting, Wireless Energy Transfer, GSM,

$I S M$,

SMS7630.

\begin{abstract}
With the expansion of wireless communication systems, the amount of signal in our environment are also increased. RF harvesting systems are designs that aims to harness these available energy in the environment. The main application of these systems, is to provide aid or extend the battery life of communication systems with low power consumption. Herein, design of a single stage RF harvester design with operation band of $1.8-2.5 \mathrm{GHz}$ had been studied. Due to the available GSM and ISM band signals, the proposed design would have good performance results. For design of rectifier stage of RF harvester, Microstrip inter-digital capacitor and microstrip stub designs had been used. With a $0 \mathrm{dBm}$ input power, the designed RF harvester had obtained output voltage of $(38 / 141) \mathrm{mV}$ with a load of $100 / 100 \mathrm{~K}$ ohm and $(125.6 / 199) \mathrm{mV}$ for load of $100 / 100 \mathrm{~K} \mathrm{ohm}$ at 1.8 and $2.4 \mathrm{GHz}$ respectively. The output impedance value of the RF harvester had been take taken between $100-100 \mathrm{k}$ ohm to evaluate the performance of the design for different load values.
\end{abstract}

Alıntı / Cite

Belen, A., Belen, M. A., (2021). GSM ve ISM Bandı RF Enerji Hasatlama Sistemleri için Tek Katı Doğrultucu Devre Tasarımı, Mühendislik Bilimleri ve Tasarım Dergisi, 9(2), 359-365.

Yazar Kimliği / Author ID (ORCID Number)

A. Belen, 0000-0001-5038-424X

M. A. Belen, 0000-0001-5588-9407

Makale Süreci / Article Process

Başvuru Tarihi / Submission Date

Revizyon Tarihi / Revision Date

Kabul Tarihi / Accepted Date

Yayım Tarihi / Published Date

14.04 .2021

22.05.2021

04.06 .2021

20.06.2021

\footnotetext{
* ilgili yazar / Corresponding author: aysu.belen@iste.edu.tr, +90-326-618-29-31
} 


\section{Giriş (Introduction)}

Günlük hayatımızda fabrikalardan sensör ağlarına, akıllı binalardan insan vücuduna kadar pek çok alanda kablosuz sensör ağları yer almaktadır. Mobil baz istasyonları, TV yayınları, el telsizleri ve cep telefonları dahil olmak üzere milyonlar ve hatta milyarlarca farklı cihazdan RF sinyal yayılmakta olup, bu sayı gün geçtikçe artan cihaz ve kullanıcı sayılarına bağlı olarak daha da artmaktadır (Paradiso, Starner, 2005), (Rebello, 2010). Burada önemli araştırmalardan biri (Cheng, Chawathe, 2005) tarafından sunulan, geniş bantlı mobil cihazların sayısının bir milyardan fazla bir sayıya ulaşması olduğu söylenebilir. Dikkat edilmesi gereken bir başka husus ise, dizüstü bilgisayar ve benzeri kablosuz Wİ-Fİ ve ISM bandında çalışan cihazların sayısının artması verilen değerlerinde gün geçtikçe artacağının belirgin bir işaretidir. Tipik bir kentsel alan konumunda bir cihaz birden fazla Wi-Fi vericisinden sinyal alabilir (Cheng, Chawathe, 2005), veya kapalı alanlardaki kamu erişim noktalarından 100mW güç seviyesindeki sinyallerin algılanması beklenen bir durumdur. Bu veriler göz önüne alındığında, düşük güçlü cihazların batarya ömrünü uzatmak ya da batarya kullanımını ortadan kaldırmak için RF enerji hasatlayıcı devrelerin kullanılabileceği belirtilmiștir (Gilbert, Balouchi, 2008; Curty, Joehl, 2000). Ortamda bulunan mevcut sinyallerin enerji kaynağı olarak dönüştürülmesini hedefleyen sistemler enerji hasatlama sistemleridir (Kasar ve ark., 2019). Hagerty ve arkadaşları tarafından 2006 yılında dizi anten yapısı ile 2 - $18 \mathrm{GHz}$ bant aralığında, RF gücünü hastalanmayı hedefleyen çalışma gerçekleștirilmiştir. Uzak alan RF enerji hasatlama çalışmalarına örnek verilmesi gereken ilginç çalışmalardan biri ise, Intel Research Seattle tarafından $960 \mathrm{KW}$ çıkış enerjisine sahip bir TV yayın istasyonu ile $4 \mathrm{~km}$ mesafedeki bir RF hasatlayıcı sisteminin performans incelemesi olmuștur (Sample, A., Smith, 2009). Başka bir benzer uzak alan RF enerji hasatlama çalışması ise, $5 \mathrm{~kW}$ çıkış gücüne sahip bir AM radyo verici istasyonu ile $2.4 \mathrm{~km}$ uzaktaki RF hasatlayıcı sisteminin performans incelemesi yapılmıștır (Ostaffe, 2010). Literatürde RF enerji hasatlama sistemleri için pek çok devre önerilmiştir. Bunlardan bir taneside Greinacher doğrultma devresidir. Bu devre düşük akım ve gerilimlere cevap verebilen şotki diyotu kullanılarak çıkış yükü üzerinde yüksek güç dönüştürme verimi ve yüksek DC gerilim elde edilmesini sağlamaktadır (Gozel ve ark., 2019), (Belen, 2018).

Düşük güçlü enerji tüketiminin yaygınlaşması sonucunda üreticiler yeni geliştirdikleri ürünlerini müşterilere düşük güç tüketen, "çok düşük güç tüketen" ve "aşırı düşük güç tüketen" diye pazarlamaktadır. Örnegin Texas Instruments'ın ürettiği 16 bit "çok düşük güç tüketen" MSP430FR597 mikroişlemcisi aktif modda yaklaşık $400 \mu \mathrm{A}$, bekleme modunda ise yalnızca $0.4 \mu \mathrm{A}$ akım çekmektedir(MSP430FR597). Bu ürünler ne kadar düşük güç tüketimine sahip olursa olsun bataryalarının doluluğunun takip edilmesi gerekmektedir. Bu aşamada enerji hasatlama devreleri ortaya çıkmıștır. Ayrıca enerji hasatlama devrelerinin kullanımı üzerine, cep telefonlarının, dizüstü bilgisayarların, tabletlerin pil ömürlerinin arttırılması üzerine çalışılmaktadır. (Liang vd., 2019; Song vd., 2019).

Bu çalışma kapsamında GSM ve ISM bandlarında kullanılmak üzere tek katlı doğrultucu devre önerilmiştir. Doğrultucu devre giriş empedansı 50ohm olarak uyumlandırma katı tasarlanmıștır. Bu katta mikroșerit saplama yapısı ve interdigital kapasitör kullanılarak $1.8 \mathrm{GHz}$ ile $2.5 \mathrm{GHz}$ arasında $-10 \mathrm{~dB}$ band genişliği elde edilmiştir. Elde edilen düşük güç seviyeleri ile kablosuz haberleşme uygulamalarında kullanılan sensörlerin, cihazların pil ömürleri uzatılabilinecektir.

\section{RF Enerji Hasatlayıcı Devre Tasarımı (RF Energy Harvester Design)}

Şekil 1'de RF enerji hasatlayıcı sistem blok şeması gösterilmiştir. Sistemin ilk bileşeni olan anten; ortamdaki elektromanyetik sinyallerin toplanması görevini görmektedir. Empedans uyumlandırma devresi doğrultucu devre ile anten girişi arasındaki empedans uyumsuzluğunu gidererek sistemdeki kayıpları önlemektedir. Doğrultulan sinyalin DC çıkış gücü yüke aktarılmadan önce DC filtre devresi tarafından filtrelenir. Tasarım aşamasında RF doğrultucu devre giriş empedansı 50ohm olacak șekilde tasarlanmıștır.

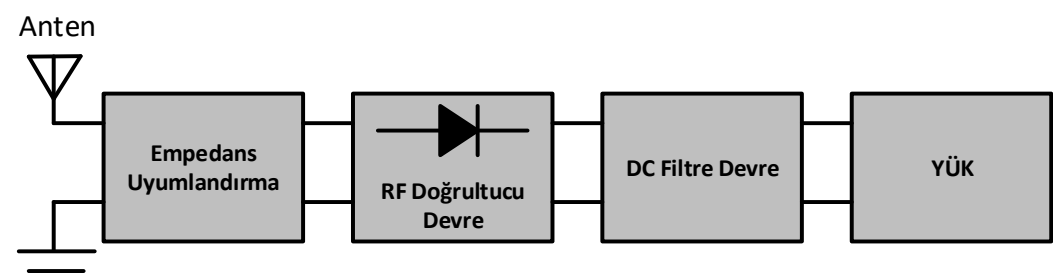

Şekil 1. RF Enerji Hasatlayıcı Blok Şeması (RF Energy Harvester Block Diagram) 


\subsection{Uyumlandırma Katı Tasarımı (Matching Stage Design)}

RF enerji hasatlayıcıların kablosuz güç iletimi sistemlerinden farkı hasatlayıcıların çok düşük güçlerle çalışmasıdır. Bu nedenle hasatlayıcıların mümkün olduğunca yüksek verimle çalıșması gerekir. Anten vasıtasıyla ortamdan alınan gücün verimli bir şekilde doğrultucuya aktarılması vazifesini uyumlandırma katı gerçekleștirmektedir. Doğrultucu devre elemanı olan diyotun doğrusal olmayan çalıșma karakteristiğinden dolayı empedans uyumsuzlukları olabilir. Bu uyumsuzlukları uyumlandırma katı kullanarak en aza indirebiliriz. Uyumlandırma katı $\mathrm{RF}$ sinyal girişinde meydana gelebilecek enerji yansımaları elimine edilerek çıkış voltajının düşmesi engellenebilecektir. Herhangi bir empedans uyumlandırma tekniği (Toplu elemanlar, Tek saplama yöntemi gibi) devre girişinde uygulanarak farklı bir frekansta rezonansa gelmesi sağlanabilir. Uyumlandırma katı tasarımında dikkat edilmesi gereken en önemli husus giriș ve çıkıș empedans değerleridir. Toplu elemanlar ile yapılan uyumlandırma katlarında uygun değerde eleman temini sorunu, sinyalde kayıp, parazitik etkiler gibi sorunlar yaşanmaktadır. Bu nedenle çalışma kapsamında RF doğrultucu devrenin uyumlandırma katında mikroşerit interdijital kapasitör ve mikroşerit saplama yapıları kullanılarak uyumlandırma sağlanmıştır.

\section{2. Şotki Diyot Seçimi (Schottky Diode Selection)}

$\mathrm{RF}$ enerji hasatlamada dikkat edilmesi gereken en önemli tasarım parametrelerinden biri diyot seçimidir. Bu tarz çalışmalarda ortamdaki enerjinin güç değerlerinin az olması nedeni ile seçilecek olan diyotlarında olabildiğince düşük açılma gerilim değeri yanı sıra, yüksek anahtarlama hızına da sahip olması gerekmektedir (H. Yan, J. G. M. Montero, 2005). Önerilen çalışma kapsamında hedeflenen 1.8 ve $2.4 \mathrm{GHz}$ frekanslarında çalışacak olan geniş bantlı doğrultucu devre tasarımı için toplu (lumped) elemanlar ile SMS-7630 schottky diyot elemanı kullanılmıştır.

Tablo 1. Şotki diyotlar ve parametreleri (Rehman ve ark., 2017). (Schottky diodes and parameters)

\begin{tabular}{|c|c|c|c|c|}
\hline Diode & $\begin{array}{c}\text { Vt } \\
\text { (V) }\end{array}$ & $\begin{array}{c}\text { Vb } \\
\text { (V) }\end{array}$ & $\begin{array}{c}\text { Rs } \\
\text { (ohm) }\end{array}$ & $\begin{array}{c}\text { Jc } \\
\text { (pF) }\end{array}$ \\
\hline HSMS-2860 & 0.65 & 7 & 6 & 0.18 \\
\hline HSMS-8202 & 0.35 & 4 & 14 & 0.26 \\
\hline SMS-7630 & 0.34 & 2 & 20 & 0.14 \\
\hline SMS7621 & 0.55 & 3 & 12 & 0.1 \\
\hline MA-40417 & 0.65 & 11 & 4.9 & 0.04 \\
\hline
\end{tabular}

Belirlenen çalışma frekansı için (1.8-5 GHz) en iyi diyot elemanını seçimi için piyasada bulunan bazı ticari ürünler incelenmiş olup tablo 1'de parametreleri ile birlikte listelenmişlerdir (Rehman ve ark., 2017). Tablodan da görüleceği üzere, en düşük Vt değeri SMS-7630 elemanı ile elde edilmektedir. Buda diyotun, diğer elemanlara kıyasla daha düşük bir giriş gücü ile aktif hale gelebileceğini göstermektedir ve bu neden ile en iyi seçim olacaktır. Bir diğer önemli parametre ise Jc diğer diyot yapılarındaki bu değerin yüksek olması elemanların performanslarının yüksek frekanslarda bozulacağını göstermektedir. Her ne kadarda (MA-40417 ve SMS-7621 elemanlarının değerleri daha iyi olsa da bu elemanların eşik değerlerinden dolayı daha yüksek bir giriş gücüne ihtiyaç duyulacaktır. Dolayısı ile çalışma kapsamında kullanılabilecek en uygun diyot yapısı SMS-7630 elemanı olarak seçilmiştir.

\subsection{Yük Empedansı Seçimi (Load Impedance Selection)}

Kablosuz haberleşme sistemlerinde kullanılan elektronik bileșenlerin belirli bir yük empedansı bulunmaktadır, bu aralıkların dışında çalışıldığında çalışma performansı düşmektedir. Bu nedenle RF doğrultucu devrelerde yük empedans aralığının seçilmesi önemli bir kriterdir. Bu çalışmada devrenin yük empedansı 4 farklı yük (100ohm, $1 \mathrm{Kohm}, 10 \mathrm{Kohm}, 100 \mathrm{Kohm}$ ) üzerinden incelenerek yük değerinin değișiminin devre performansına etkisi gözlenmiştir.

\subsection{Doğrultucu Tasarımı (Rectifier Design)}

Kablosuz haberleşme sistemlerinin yaygınlaşmasıyla ortamlarda pek çok frekansta sinyaller bulunmaktadır. Ortamda bulunan mevcut sinyallerin enerji kaynağı olarak dönüştürülmesini hedefleyen tek katlı doğrultucu devrenin gösterimi şekil 2'de verilmiştir. Şekil 2'de D1 ve D2 zero-bias low barrier schottky diyotları kullanılarak düşük güçlü RF sinyallerin yüksek çıkış gerilimi ile toplanması sağlanmıștır. Devrenin girişinde $50 \mathrm{ohm}$ giriş empedansına göre uyumlandırma katı eklenmiștir. Uyumlandırma katı antenin doğrultucu katına maksimum güç aktarmasında önem taşımaktadır. Bir enerji hasatlama devresinde çalışma performansının değerlendirildiği parametreler; çalışma frekansı, toplam güç dönüştürme verimi ve yük direncine göre çıkış gerilimidir (Gozel ve ark., 2019), (Kasar ve ark., 2019). 


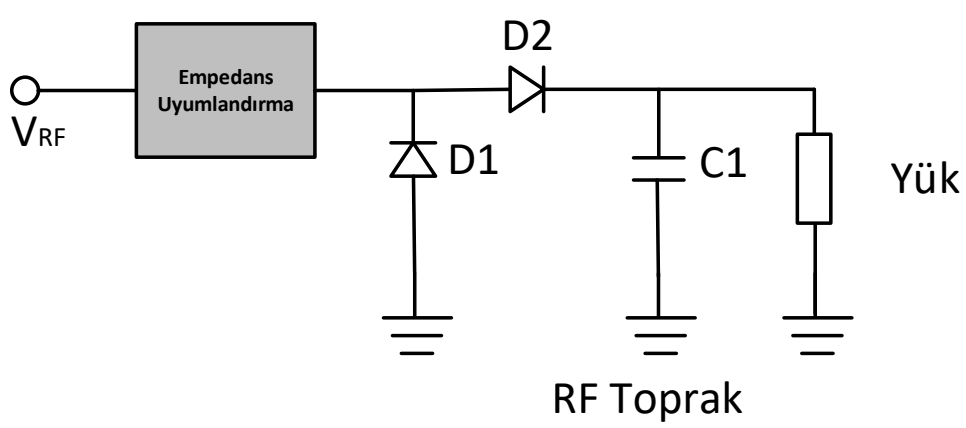

Şekil 2. Tek Katlı Tam Dalga Doğrultucu (Single Stage Full Wave Rectifier)

$1.8 \mathrm{GHz}$ (GSM) ve 2.4(ISM) bandlarını kapsayan geniş bandlı tek katlı doğrultucu devre tasarımı bu çalıșma kapsamında önerilmiştir. $+30 \mathrm{dBm}$ ile $-30 \mathrm{dBm}$ arasında devreye giriş gücü uygulanarak yük üzerinden alınabilecek güç seviyesi izlenmiștir.

Şekil 3'te uyumlandırılmış devrenin şematik devre gösterimi verilmiştir. D1 diyot modeli olarak SMS7630, C1 kapasite değeri 100pF kullanılmıştır. Devre boyutları 45mmx30mm dir.

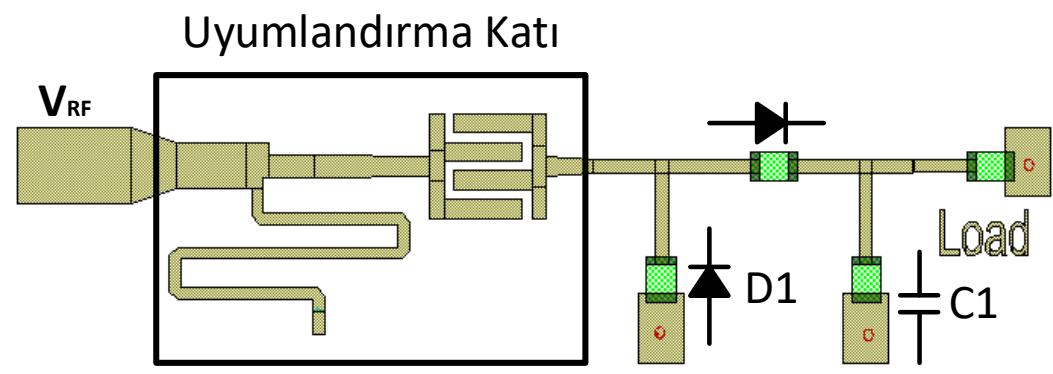

Şekil 3. Tek Katlı Tam Dalga Doğrultucusu Baskı Devre Gösterimi(Single Stage Full Wave Rectifier Printed Circuit)

Tablo 2. Uyumlandırma katı parametre listesi (Matching stage parameter list)

\begin{tabular}{|c|c|c|c|}
\hline L1 & $5.14 \mathrm{~mm}$ & W1 & $4.58 \mathrm{~mm}$ \\
\hline L2 & $10.5 \mathrm{~mm}$ & $\mathbf{W 2}$ & $0.88 \mathrm{~mm}$ \\
\hline $\mathbf{W P}$ & $0.65 \mathrm{~mm}$ & $\mathbf{W 3}$ & $2.25 \mathrm{~mm}$ \\
\hline S & $0.4 \mathrm{~mm}$ & $\mathbf{W 4}$ & $0.5 \mathrm{~mm}$ \\
\hline
\end{tabular}

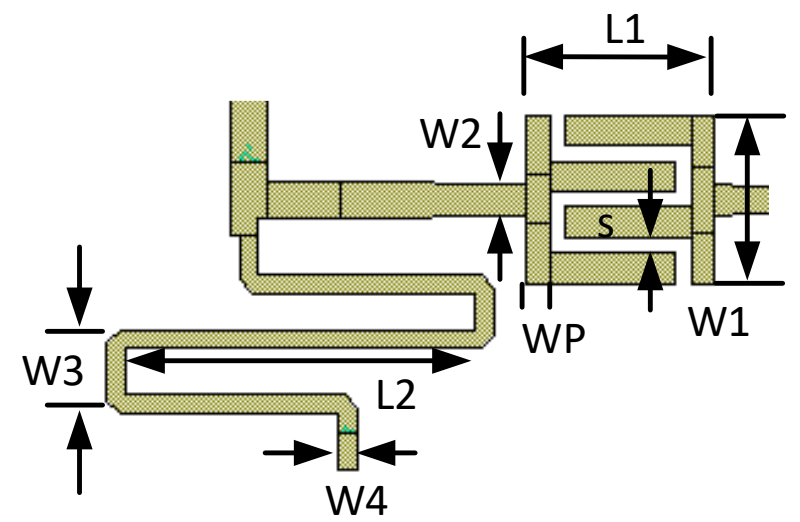

Şekil 4. Geniş Band Uyumlandırma Devresi (Wideband Matching Circuit)

Şekil 4'teki devre yapısında uyumlama devresi tasarlanmış, bu uyumlama devresi interdigital kapasitör ve mikroşerit saplama elemanlarını kapsamaktadır. Uyumlandırma katı değișken parametreleri tablo 2'de verilmiștir. Şekil 5'te devreye ait geri-dönüș $\left(S_{11}\right)$ kaybı verilmiș olup, görüleceği üzere, hedeflenen çalıșma bant aralığında $-10 \mathrm{~dB}$ den daha az bir değere sahiptir. $1.75 \mathrm{GHz}$ ile $2.45 \mathrm{GHz}$ arasında $-10 \mathrm{~dB}$ band genişliği elde edilmiştir. 


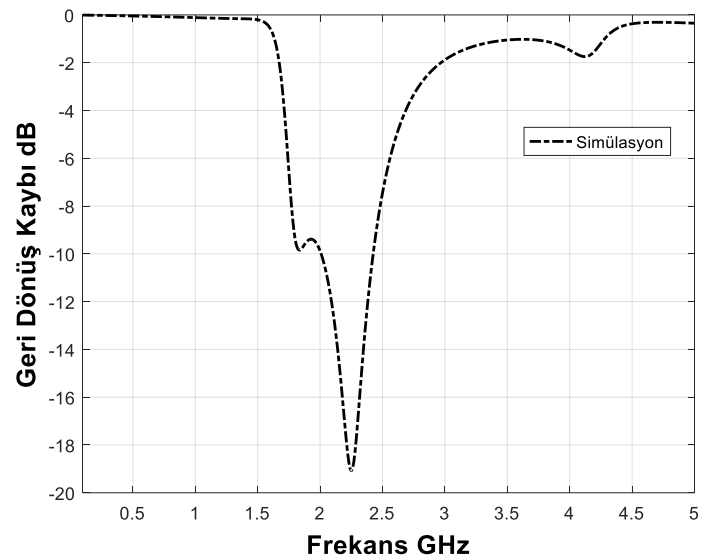

Şekil 5. Uyumlandırılmış tek katlı devrenin geri dönüş kaybı( Return Loss of Matched Single Stage Circuit)

Şekil 3'te verilen devrenin yük direnci $(100 \mathrm{ohm}, 1 \mathrm{Kohm}, 10 \mathrm{Kohm}$ ve $100 \mathrm{Kohm})$ değiştirilerek çıkışta elde edilebilecek çıkış güçleri Tablo 3 ve 4'te verilmiştir. Düşük yük direncinde elde edilen çıkış gücünün daha yüksek olduğu gözlenmiştir. Doğrultucu devrede düşük yük direncinde verim yüksek olmasına rağmen çıkışta elde edilen gerilim değeri düşük seviyededir.

Tablo 3. 100 ohm ile 100Kohm Arasında Giriș Gücüne Göre Çıkıș Gücü 1.8GHz (Variation of output power with respect to input power for different loads $(100-100 \mathrm{k} \Omega)$ )

\begin{tabular}{|c|c|c|c|c|}
\hline \multirow{2}{*}{$\begin{array}{c}\text { Giriş } \\
\text { Gücü } \\
\mathbf{d B m}\end{array}$} & \multicolumn{4}{|c|}{ Çıkış Gücü dBm } \\
\cline { 2 - 5 } & $\mathbf{1 0 0 0 h m}$ & $\mathbf{1 K o h m}$ & $\mathbf{1 0 K o h m}$ & $\mathbf{1 0 0 K o h m}$ \\
\hline $\mathbf{- 3 0}$ & -40.7 & -48.5 & -58.4 & -68.4 \\
\hline $\mathbf{- 2 5}$ & -35.7 & -43.6 & -53.5 & -63.3 \\
\hline $\mathbf{- 2 0}$ & -30.7 & -38.4 & -48.34 & -58.2 \\
\hline $\mathbf{- 1 5}$ & -25.5 & -33.5 & -43.12 & -52.8 \\
\hline $\mathbf{- 1 0}$ & -20.8 & -28.4 & -37.8 & -47.3 \\
\hline $\mathbf{- 5}$ & -15.6 & -23.2 & -32.5 & -42 \\
\hline $\mathbf{0}$ & -10.8 & -18.4 & -27.5 & -37.1 \\
\hline $\mathbf{5}$ & -5.6 & -13.4 & -22.63 & -32.5 \\
\hline $\mathbf{1 0}$ & -0.6 & -8.4 & -17.8 & -27.8 \\
\hline $\mathbf{1 5}$ & 4.3 & -3.5 & -13.1 & -23.1 \\
\hline $\mathbf{2 0}$ & 9.4 & 1.6 & -8.3 & -18.2 \\
\hline $\mathbf{2 5}$ & 14.3 & 6.5 & -3.33 & -13.3 \\
\hline $\mathbf{3 0}$ & 19.3 & 11.5 & 1.63 & -8.4 \\
\hline
\end{tabular}

Tablo 4. 100 ohm ile 100Kohm Arasında Giriş Gücüne Göre Çıkış Gücü 2.4GHz (Output power @ 2.4 GHz for variant loads ( $100-100 \mathrm{k} \Omega$ ))

\begin{tabular}{|c|c|c|c|c|}
\hline \multirow{2}{*}{$\begin{array}{c}\text { Giriş } \\
\text { Gücü } \\
\mathbf{d B m}\end{array}$} & \multicolumn{4}{|c|}{ Çıkış Gücü dBm } \\
\cline { 2 - 5 } & $\mathbf{1 0 0 0 h m}$ & $\mathbf{1 K o h m}$ & $\mathbf{1 0 K o h m}$ & $\mathbf{1 0 0 K o h m}$ \\
\hline $\mathbf{- 3 0}$ & -38.1 & -44.8 & -54.6 & -64.57 \\
\hline $\mathbf{- 2 5}$ & -33.6 & -39.8 & -49.6 & -59.5 \\
\hline $\mathbf{- 2 0}$ & -28.1 & -34.8 & -44.5 & -54.5 \\
\hline $\mathbf{- 1 5}$ & -23.6 & -29.79 & -39.5 & -49.3 \\
\hline $\mathbf{- 1 0}$ & -18 & -24.77 & -34.3 & -44.12 \\
\hline $\mathbf{- 5}$ & -13 & -19.75 & -29.2 & -39 \\
\hline $\mathbf{0}$ & -8 & -14.7 & -24.2 & -34 \\
\hline $\mathbf{5}$ & -3.1 & -9.7 & -19.3 & -29 \\
\hline $\mathbf{1 0}$ & 1.96 & -4.7 & -14.36 & -24.3 \\
\hline $\mathbf{1 5}$ & 7 & 0.25 & -9.4 & -19.4 \\
\hline $\mathbf{2 0}$ & 12 & 5.25 & -4.5 & -14.5 \\
\hline $\mathbf{2 5}$ & 17 & 10.24 & 0.5 & -9.5 \\
\hline $\mathbf{3 0}$ & 22 & 15.23 & 5.5 & -4.5 \\
\hline
\end{tabular}

Tablo 5'de SMS7630 diyotlu tek katlı doğrultucu devre tasarımı iki farklı yük direnci için elde edilebilecek DC gerilim seviyesi verilmiştir. $-30 \mathrm{dBm}$ ile $+30 \mathrm{dBm}$ arasında RF giriş uygulanarak çıkış gerilimi elde edilmiştir. Elde edilen sonuçlara göre tek katlı doğrultma devresinin düșük yük direncinde verimi yüksek olduğu, yüksek yük 
direncinde ise çıkış voltajının yüksek olduğu gözlenmiştir. Buradan RF doğrultucu devrenin kullanılacağı uygulamaya göre yük direnci seçiminin önemi anlaşılmaktadır.

Tablo 5. RF Giriş güç - DC Çıkış gerilim (PIN-VOUT) (Input RF power - DC output voltage values)

\begin{tabular}{|c|c|c|c|c|}
\hline $\begin{array}{c}\text { Giriş RF } \\
\text { Gücü } \\
(\mathbf{d B m})\end{array}$ & \multicolumn{4}{|c|}{ Vout (mV) (DC) } \\
\cline { 2 - 5 } & $\begin{array}{c}\mathbf{1 0 0 0 h m} \\
(\mathbf{1 . 8 G H z})\end{array}$ & $\begin{array}{c}\mathbf{1 0 0 K o h m} \\
(\mathbf{1 . 8 G H z})\end{array}$ & $\begin{array}{c}\mathbf{1 0 0 0 h m} \\
\mathbf{( 2 . 4 G H z )}\end{array}$ & $\begin{array}{c}\mathbf{1 0 0 K o h m} \\
(\mathbf{2 . 4 G H z})\end{array}$ \\
\hline $\mathbf{- 2 0}$ & 9.2 & 12.2 & 12.5 & 18.8 \\
\hline $\mathbf{- 1 0}$ & 28.9 & 43.1 & 39.7 & 62.2 \\
\hline $\mathbf{0}$ & 38 & 141 & 125.6 & 199 \\
\hline $\mathbf{1 0}$ & 294 & 407.4 & 396.2 & 608 \\
\hline $\mathbf{2 0}$ & 932 & 1228.8 & 1261 & 1871 \\
\hline $\mathbf{3 0}$ & 2910 & 3800 & 3974.9 & 5950 \\
\hline
\end{tabular}

Tablo 6'da tasarlanan doğrultucu devrenin performansı literatürdeki çalışmalarla kıyaslanmıştır. Elde edilen sonuçlara göre, doğrultucu devre tasarımı ile düşük empedans seçiminde veriminin daha yüksek, yüksek empedans seçiminde ise çıkış geriliminin arttığı gözlenmiştir. Bu konuda yapılacak iyileştirme çalışmalarında kullanılacak sistem yükünün belirlenerek devrenin çıkış gerilimi arttırılabilir.

Tablo 6. Doğrultucu Performans Karşılaştırması (Comparison of Rectifier Performances)

\begin{tabular}{|c|c|c|c|c|c|}
\hline Kaynak & $\begin{array}{c}\text { Bu } \\
\text { Calışmada }\end{array}$ & $\begin{array}{c}\text { (Papotto, } \\
\text { Carrara, 2011) }\end{array}$ & $\begin{array}{c}\text { (Le, } \\
\text { Mayaram, } \\
\mathbf{2 0 0 8}\end{array}$ & $\begin{array}{c}\text { (Kasar ve ark., } \\
\text { 2019). }\end{array}$ & $\begin{array}{c}\text { (Liu ve ark., } \\
\text { 2019). }\end{array}$ \\
\hline $\boldsymbol{f ( G H z )}$ & $1.8 / 2.4$ & 0.9 & 0.91 & 2.45 & 2.1 \\
\hline $\begin{array}{c}\mathbf{P}_{\mathbf{R F}} \\
(\mathbf{d B m})\end{array}$ & 10 & -22 & -15 & 9 & 0 \\
\hline $\mathbf{V}_{\text {rec }}(\mathbf{V})$ & $0.4 / 0.6$ & 1 & 2.8 & 1 & 0.8 \\
\hline Kademe & 1 & 17 & 16 & 1 & 2 \\
\hline Teknoloji & SMS7630 & $\begin{array}{c}\text { CMOS } \\
90 \mathrm{um}\end{array}$ & $\begin{array}{c}\text { CMOS } \\
150 \mathrm{um}\end{array}$ & HSMS-285C & SMS7630 \\
\hline Boyut & $45 \mathrm{~mm} \times 30 \mathrm{~mm}$ & $1.31 \mathrm{~mm} \times 1.03 \mathrm{~mm}$ & --- & $67.4 \mathrm{~mm} \times 11.2 \mathrm{~mm}$ & $40 \mathrm{~mm} 25 \mathrm{~mm}$ \\
\hline
\end{tabular}

\section{Sonuç ve Tartışma (Result and Discussion)}

$\mathrm{Bu}$ çalışma kapsamında GSM ve ISM bandlarında kullanılmak üzere tek katlı doğrultucu devre önerilmiştir. Doğrultucu devre giriş empedansı 50ohm olarak uyumlandırma katı tasarlanmıştır. Bu katta mikroşerit saplama yapısı ve interdigital kapasitör kullanılarak $1.8 \mathrm{GHz}$ ile $2.5 \mathrm{GHz}$ arasında $-10 \mathrm{~dB}$ band genişliği elde edilmiştir. Yapılan simülasyon çalışmalarında yük direnci değerinin değeri değiştirilerek doğrultucu verimi ve çıkış voltajı değişimi incelenmiştir. Tek katlı doğrultma devresinin düşük yük direncinde verimi yüksek olduğu, yüksek yük direncinde ise çıkış voltajının yüksek olduğu gözlenmiştir. Buradan RF doğrultucu devrenin kullanılacağı uygulamaya göre yük direnci seçiminin önemi anlaşılmaktadır. $10 \mathrm{dBm}$ giriş gücünde $1.8 \mathrm{GHz}$ de $100 \mathrm{ohm}$ yük direncinde $294 \mathrm{mV}$ iken $100 \mathrm{Kohm}$ yük direncinde $407 \mathrm{mV}$ ve $2.4 \mathrm{GHz}$ de $100 \mathrm{ohm}$ yük direncinde $396 \mathrm{mV}$ iken $100 \mathrm{Kohm}$ yük direncinde $608 \mathrm{mV}$ çıkış gerilimi elde edilmiştir. Elde edilen düşük güç seviyeleri ile kablosuz haberleşme uygulamalarında kullanılan sensörlerin, cihazların pil ömürleri uzatılabilir.

\section{Çıkar Çatışması (Conflict of Interest)}

Yazarlar tarafından herhangi bir çıkar çatışması beyan edilmemiştir. No conflict of interest was declared by the authors.

\section{Kaynaklar (References)}

Belen, M.A. 2018. RF Enerji Hasatlama Sistemleri için Çift Bandli Greinacher Doğrultucu Devre Tasarimi. Mühendislik Bilimleri ve Tasarım Dergisi 6, 2, 348-353.

Cheng, Y.-C., Chawathe, Y., LaMarca, A., Krumm, J., 2005. Accuracy Characterization for Metropolitan-Scale Wi-Fi Localization. Proc. Third Int. Conf. on Mobile Systems, 233-245.

Curty, J.-P., Joehl, N., Krummenacher, F., Dehollain, C., Declercq, M., 2000. A model for m-power rectifier analysis and design. IEEE Trans. Circuits Syst. I, 52 (12), 2771-2779.

Gilbert, J.M., Balouchi, F., 2008. Comparison of energy harvesting systems for wireless sensor networks. Int. J. Autom. Comput., 5 (4), 334-347. 
Gozel, M. A., Kahriman, M., Kasar, O. (2019). Design of an Efficiency-Enhanced Greinacher Rectifier Operating in the GSM 1800 Band by using Rat-Race Coupler For RF Energy Harvesting Applications. International Journal of RF and Microwave Computer-Aided Engineering, 29 (1), 1-8.

Hagerty, J.A., Helmbrecht, F.B., McCalpin, W.H., Zane, R., Popovic, Z.B., 2004. Recycling ambient microwave energy with broadband rectenna arrays. IEEE Trans. Microw. Theory Tech., 52(3), 1014-1024.

Kasar, O., Kahriman, M., Gozel, M. A. (2019). Application of Ultra Wideband RF Energy Harvesting by Using Multisection Wilkinson Power Combiner. International Journal of RF and Microwave Computer-Aided Engineering, 29 (1), 1-8.

Kasar, O , Kahriman, M , Gözel, M . (2019). Wilkinson Güç Birleştirici Kullanarak İki Girişli RF Enerji Hasatlama Devresi ve DC Yük Analizi. Mehmet Akif Ersoy Üniversitesi Fen Bilimleri Enstitüsü Dergisi, 10 (1), 68-72.

Le, T., Mayaram, K., Fiez, T., 2008. Efficient Far-Field Radio Frequency Energy Harvesting For Passively Powered Sensor Networks. IEEE J. Solid-State Circuits, 43 (5), 1287-1302.

Liang, X., Zhao, T., Jiang, W., Yu, X., Hu, Y., Zhu, P., Zheng, H., Sun, R., Wong, C.-P. (2019). Highly Transparent Triboelectric Nanogenerator Utilizing in-situ Chemically Welded Silver Nanowire Network as Electrode for Mechanical Energy Harvesting and Body Motion Monitoring. Nano Energy, 59, 508-516.

Liu, W., Huang, K., Wang, T.,Zhang, Z., Hou, J., (2020). A Broadband High-Efficiency RF Rectifier for Ambient RF Energy Harvesting," IEEE Microw.e Wirel. Comp. Lett., vol. 30, no. 12, pp. 1185-1188, Dec. 2020, doi: 10.1109/LMWC.2020.3028607.

MSP430FR597x(1), MSP430FR587x(1) Mixed-Signal Microcontrollers $\quad$ (Rev. http://www.ti.com/lit/ds/slase66b/slase66b.pdf, (Accessed on 05/04/2021).

Olgun, U., Chen, C.-C., Volakis, J.L., 2012. Design of an Efficient Ambient WiFi Energy Harvesting System. IET Microw. Antennas Propag., 6(11), 1200-1206.

Ostaffe, H., 2010. Power out of Thin Air: Ambient RF Energy Harvesting for Wireless Sensors. http://powercastco.com/PDF/Power-Out-of-Thin-Air.pdf

Papotto, G., Carrara, F., Palmisano, G., 2011. A 90-nm CMOS Threshold-Compensated RF Energy Harvester, Solid-State Circuits. IEEE Journal Solid-State Circuits , 46(9), 1985 - 1997.

Paradiso, J.A., Starner, T., 2005. Energy Scavenging for Mobile and Wireless Electronics. IEEE Pervasive Comput., 4 (1), $18-27$.

Rebello, J., 2010. Global Wireless Subscriptions Reach 5 Billion. Available: http://www.isuppli.com/Mobile-and-Wirelesscommunications/News/Pages/Global-Wireless-Subscriptions-Reach-5-Billion.aspx

Rehman, M. U., Ahmad W., Qureshi, M. I., Khan, W. T. 2017. A Highly Efficient Tri Band (GSM1800, WiFi2400 and WiFi5000) Rectifier for Various Radio Frequency Harvesting Applications, 2017 Progress in Electromagnetics Research Symposium Fall (PIERS - FALL), 2017, 2039-2044.

Sample, A., Smith, J, 2009. Experimental Results with Two Wireless Power Transfer Systems. Proc. IEEE RWS, 16-18.

Song, G. J., Kim, K.-B., Cho, J. Y., Woo, M. S., Ahn, J. H., Eom, J. H., Ko, S. M., Yang, C. H., Do Hong, S., Jeong, S. Y. 2019. Performance of a Speed Bump Piezoelectric Energy Harvester for an Automatic Cellphone Charging System. Applied Energy, 247, 221227.

Yan, H., Montero, J. G. M., Akhnoukh, A., de Vreede, L. C. N., Burghart, J. N., 2005. An integration Scheme for RF Power Harvesting. 8th Annu. Workshop Semiconductor Advances Future Electron. Sensors, Veldhoven, the Netherlands. 